\title{
The application of UAV images in flood detection using image segmentation techniques
}

\author{
N. S. Ibrahim ${ }^{1}$, S. M. Sharun², M. K. Osman ${ }^{3}$, S. B. Mohamed ${ }^{4}$, S. H. Y. S. Abdullah \\ 1,2,4,5 Faculty of Innovative Design and Technology, Universiti Sultan Zainal Abidin (UniSZA), \\ Gong Badak Campus, Terengganu, Malaysia \\ ${ }^{3}$ Faculty of Electrical Engineering, UiTM, Permatang Pauh Campus, Pulau Pinang, Malaysia
}

\begin{tabular}{l} 
Article Info \\
\hline Article history: \\
Received Sep 22, 2020 \\
Revised Jun 26, 2021 \\
Accepted Jul 3, 2021 \\
\hline
\end{tabular}

\section{Keywords:}

Flood detection

K-mean clustering

Region growing segmentation

Segmentation technique

Unmanned arial vehicle

\begin{abstract}
The application of unmanned aerial vehicle (UAV) used to capture the images of the flood areas are becoming interest of most researchers recently. This is due to its versatilities of capturing the images with low-cost and real time responses. At present, the captured images are analysed manually by human experts, which cause the task labourous, time consuming and prone to error. This study aims to develop an UAV-based automated flood detection system. Samples of images that consist of land and river areas were capture using a camera attached to UAV to emulate flooded and non-flooded areas. The RGB and HSI colour models were utilised to represent the flood images. Two image segmentation methods were studied, which are k-mean clustering and region growing. The segmented images were validated with manually segmented (ground truth) images. Simulation results show that the RG using gray images gave better segmentation accuracy (88\%) as compared to the Kmean clustering (76\%). Finally, an automated flood monitoring system based on the region growing method, called flood detection structure (FDS) was developed to detect and analyse the flood severity.
\end{abstract}

This is an open access article under the CC BY-SA license.

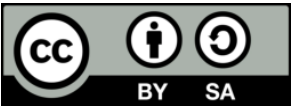

\section{Corresponding Author:}

Siti Maryam Sharun

Faculty of Innovative Design and Technology

Universiti Sultan Zainal Abidin

Kampus Gong Badak, 21300 Kuala Nerus, Terengganu, Malaysia

Email: sitimaryam@unisza.edu.my

\section{INTRODUCTION}

Flood is a natural disaster that often happens, especially in urban or complex area. This situation can be due to natural phenomenal or rapid development without mitigation that causes changes in the structure of the earth's surface then further damages the hydrology systems. It gives a significant impact in the social life, economics and development. Monsoon flood, also known as seasonal flood is the common phenomenon that is most feared especially for the east coast residents. A huge concern regarding to the number of deaths related to flood since it is the highest compared to other incidents such as earthquake and hurricane combined that is rarely happens in Malaysia [1].

The department of irrigation and drainage (DID) is a government agency responsible for the flood monitoring in Malaysia. The agency had developed a website called Infobanjir to monitor, alert and give information to the citizen regarding the flood situation. The agency has integrated information obtained from closed-circuit television (CCTV) camera, manual stick gauge and telemetry for flood monitoring. However, this system is not able to monitor any overflowing of river water (danger stage). Even though there is constant monitoring using the webcam, the application of the webcam itself is still limited. The CCTV is 
often placed at the flood-raging area marked with a stick gauge to monitor the water level. The memory of the CCTV lasted for 25 days and automatically delete when surpassed.

Satellite surveillance data have been used as an alternative for detection and monitoring flood areas [2]-[5]. It is a sophisticated technology that able to monitor disaster in huge coverage areas. However, the application of this satellite is not available on the peak disaster situation. Moreover, images obtained from a satellite require further processes in order to validate their information. Generally in detection process based on maps or images, it can be complete with specifically on appearance ahead of time [6].

Recent study shows that UAV can be used to observe the areas that is unreachable, focused area, loss of communications and complex urban sites [7]. It is frequently used to provide the real situations with real colour images through visual interpretation and provide information of the inundation areas. The images may be implemented for mapping [8], characterizing and also monitoring of the floods situations. Even though the research about UAV in flood monitoring is still infancy stages [1], [7], [9], but it is a good platform to transforms this technologies in attempt to create a smart cities in current trends and also gives the best benefits with fast responses from the images especially to the rescue teams.

Image processing techniques such as k-mean clustering and region growing have been applied in many remote sensing applications. K-mean is a clustering method that groups dataset into predefined number of disjoint point, which know as clusters [10]. Region growing is a semi-supervised segmentation technique that perform segmentation based on the similary between seed and the neighbourhood pixels to organize its region. Both methods are widely used in disaster detection, clouds conditions, and agriculture [11], [12]. Other segmentation methods such as complex image processing, digital surface model and decision fusion of neural networks were also used with UAV for disaster damage assessment, field measurement of water level, three-dimensional inundation mapping and remote estimation of flooded areas [13]-[19]. Therefore, segmentation methods have the potential to be used in the flood disaster monitoring system.

This study presents an alternative method of flood detection using UAV images. The algorithm was created based k-mean clustering and region growing in segmentation process, to identify the difference between flooded and non-flooded images from the values of pixels. The algorithm created to segment and classifies the images into flooded or non-flooded images, based on quantitative evaluation of the images that were captured using UAV with a fixed flying altitude.

\section{RESEARCH METHODOLOGY}

This section presents the proposed methodology to automate the flood detection process using image clustering methods. An overview of the process is shown in Figure 1. The method consists of three main parts which are image pre-processing, image segmentation and development of flood detection structure (FDS). Each part will be discussed in detail in the following sections.

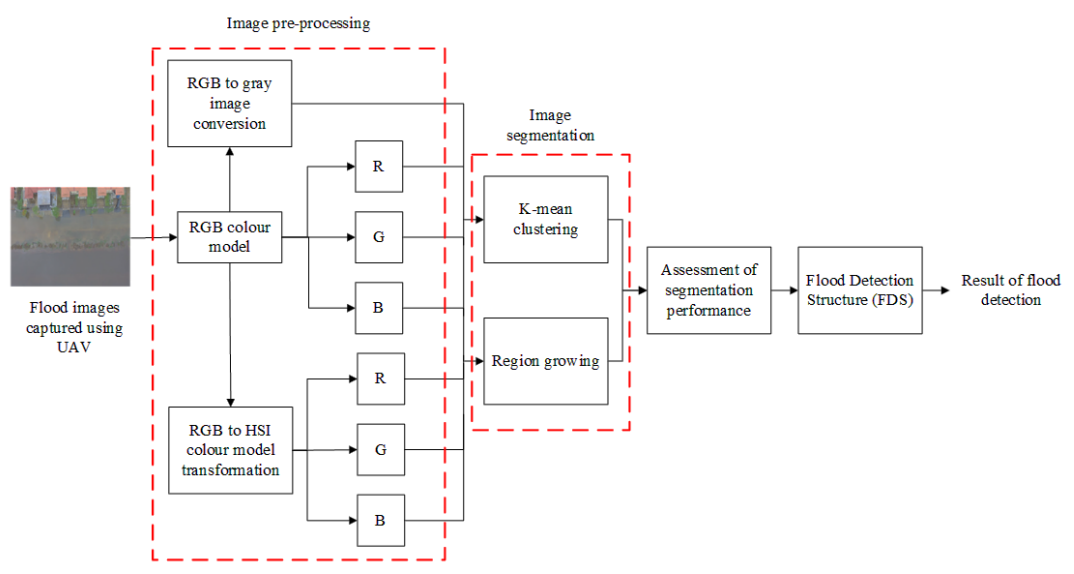

Figure 1. Overview of the proposed methodology for automatic flood detection system using UAV images

\subsection{Study area}

Kuala Nerus is a complex residential area with high number of population since it is situated nearby to the two public universities in Terengganu which are Universiti Malaysia Terengganu (UMT) and Universiti Sultan Zainal Abidin (UniSZA) [20]. The focused study area of this research is Taman Desa Tanjung Damai, a complex residential area in Kuala Nerus, Terengganu, Malaysia. This location was 
selected due to great exposure of flood especially during the monsoon season. Based on the estimated data from Department of Social Welfare (JKM), 674 victims were placed at the flood relief centre in 2017 which was the highest number of victims on the area since 2014 . The focused area has been heavily affected by flood since the area was developed back in 2008.

\subsection{Image acquisition and pre-processing}

To monitor the flood-prone areas, the mini aircraft UAV DJI Phantom 3 has been used in this research. In order to make predictions about the impact of certain quantities of rainfall that caused flood, the runoff data must be considered within a hydrographical model from the areas that often flooding and areas was affected by flood changes. The flight altitude for UAV was set as $150 \mathrm{~m}$. The UAV mini aircraft was equipped with a 12-megapixel digital camera and has flight time up to 25 minutes. The UAV captures random areas that exposed to flood. The high-resolution camera was transformed to DJI Go application in the smart phone, to download the images to the controller. In this study, a total of 30 images of flooded area were captured for the analysis.

\subsection{Color model transformation}

Two colour models, RGB and HSI were used to represent the UAV flood images. On top of that, the RGB colour image was also converted to gray-level image and compared with both RGB and HSI models. The equations for RGB to HSI colour transformation can be found in [21]. Meanwhile, the 'rgb2gray' function in MATLAB is used to convert RGB images to gray-level image.

\subsection{Image segmentation method}

Two segmentation methods were applied in this study including region growing and K-mean clustering techniques. K-mean clustering is the technique to identify numerical clustering algorithm. The main objective of the process is to determine natural grouping of a set of patterns, points, or objects in the images or data [10]. K-means approach will be used in order to cluster the images since it is simple and fast method yet easy to implement. K-means is one of the simplest algorithms that solve the clustering problem [22]. K-Mean clustering was first assigned to an arbitrary initial cluster vector to generate each pixel to the nearest centre point cluster. Then, the new cluster mean vectors were calculated based on all the pixels in one cluster. In this case, the initialization cluster value was set to $k=2$ in order to ensure the cluster separately into 2 class. The mainly proposed of this segmentation process and scope information consist of three (3) parts:

\subsubsection{Image segmentation using k-mean clustering}

1) Selection of $k$ point based on the fixed value of cluster $k=2$ to represented by pixel value that similarity data to the nearest centre

2) The algorithm looping to recalculate the new centre position and continuously repeated until all pixels are selected.

3) The groupings are based on the most minimum nearest neighbours. Each representing value of the selected centre is dissimilar.

Meanwhile, in the Region Growing technique the value pixel of neighbour or centre point of similar properties were created into larger values regions. It begins with a seed pixel in an image and will grow into regions by iteratively adding unassigned neighbouring pixels that satisfy some homogeneity criterion with the existing region of the seed pixel [23].

\subsubsection{Image segmentation using region growing}

1) Select the seed point in binary image pixels. Assume the initial seed to the first pixel is iteratively merged according to the same constraints.

2) Choose the arbitrary seed point and make comparison with the neighbours.

3) Region Growing grows by adding similar pixels; then the size of region also grows.

4) When one region stops, the RG chooses another seed pixel which is not yet belong to any region then repeat the process $(\mathrm{C})$.

5) The entire process was completed when all the pixels belong to same region.

For region growing process, the algorithm set up manually in range between (0.2-0.5) as accordingly tolerances based on the values of each colour model transformation. This estimation value obtained based on several experimental on previous study. Otherwise, in this case the image not able to be segmented if applied the tolerances value less than 0.1 and more than 0.5 . However, this tolerances value is depending on the characteristic of image itself. The proposed segmentation process applied to determine the best quality assessments result by applying different colour models which are RGB, HSI and grey scale 
images. Initially, the original RGB colour images were converted into HSI colour model. Then, each colour component (R, G, B, H, S, I) in the colour model were improved by improving (I+S) and (I-S) prior to segmentation process. Similarly, the intensity value of grey scale images was also extracted for comparison with the colour model [24].

\subsubsection{Performance assessment for image segmentation}

This classified images were analysed and compared on a pixel-by-pixel basic to detect any changes [25]. Proposed algorithms based on K-mean clustering and Region Growing were used to detect flood extents for calibrating and validating an urban flood inundation model in an offline situation. Quantitative analysis was conducted by evaluating the accuracy and precision of the segmented UAV images by using the following (1) and (2), respectively.

$$
\begin{aligned}
& \text { Accuracy }(\%)=\left(\frac{T P+T N}{T P+F P+T N+F N}\right) \times 100 \\
& \text { Precision }(\%)=\left(\frac{T P}{T P+F N}\right) \times 100
\end{aligned}
$$

where, TP is the true positive, TN is the true negative, FP represents the false positive and FN represents the false negative.

\subsection{Flood detection structure (FDS)}

To demonstrate the potential of the proposed method in detecting flood areas, image segmentation algorithm with the highest average accuracy performance of segmentation and highest performance colour component will select to use in flood detection structure (FDS). The formulation of FDS is highlighted in (3).

$$
F D S=\frac{\text { no of pixel associated with flood }}{\text { total no of pixel in the image }} \times 100
$$

In basic segmentation process, the references are based on supervised segmentation which is manually classification process [26]. Different focused point will produce different result and the result will be affected by various factors such homogeneity, content and texture. Such in study [27], used the ground truth as gold standard in attempt to validate their segmentation performance in skin detection. The ground truth process is a manually segmented that consider as fully segmented whereby $100 \%$ accurate with human validation in inferring their relation support with segmented and real images.

So that, as refers to study [6], it will be considers that if the segmented performance value is less than $50 \%$ then the images will be classes as non-flooded. This formulate is based on evaluation of nonoverlapped patches with the dimension of $50 \times 50$ pixels in each image. All the analysis was conducted using MATLAB software.

\section{RESULT AND DISCUSSION}

A total of 30 images of flooded area were captured for this study. Figure 2 shows an example of flood image that was captured using the UAV. Initial observation showed that the whole area was covered with water. The images were decomposed and segmented for further analysis.

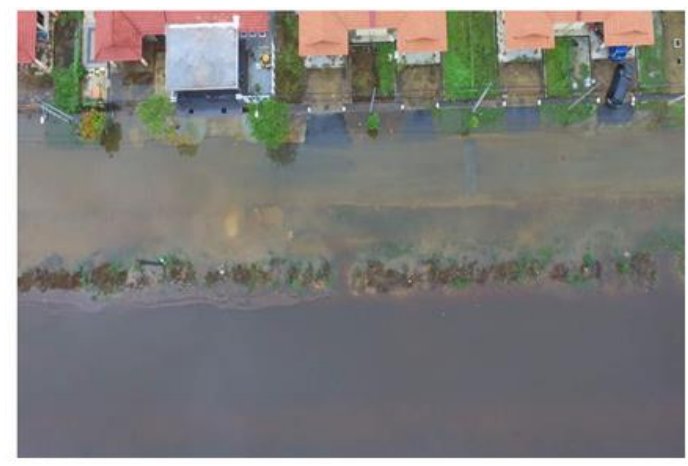

Figure 2. Flooded residential area captured by UAV 
Figure 3 visualises each colour channel of RGB as shown in Figure 3(a)-(c) and HSI as shown in Figure 3(d)-(f), as well as the gray level image as shown in Figure 3 (i). For the improvement of the S and I of HSI, the study also proposed two combinations of colour channels which are I+S and I-S, as depicted in Figures 3(g) and 3(h), respectively. However, further analysis is conducted only on the $\mathrm{I}+\mathrm{S}$ colour composition poor image visualization for I-S composition. The I+S composition provides better separation of the water and ground in the images. On the other hand, the I-S composition was unable to differentiate between the wet and land areas in the images. Similar result has been reported by [28] where the intensity value of an image reduced when the saturation value decreased.

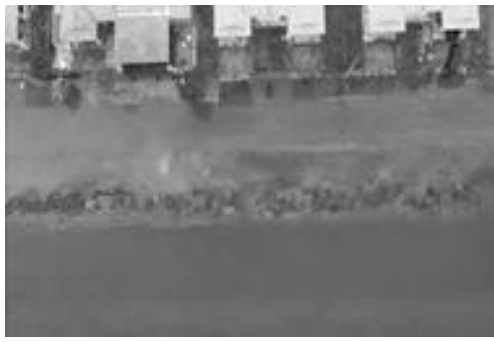

(a)

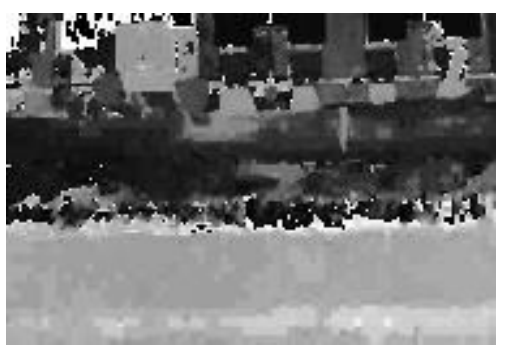

(d)

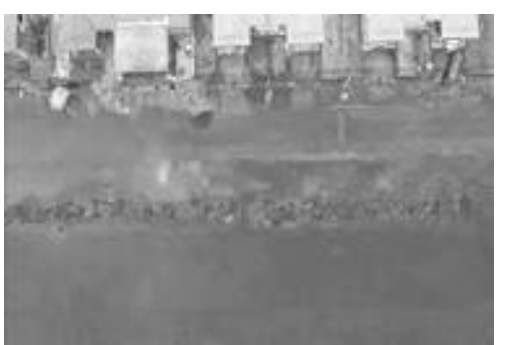

(g)

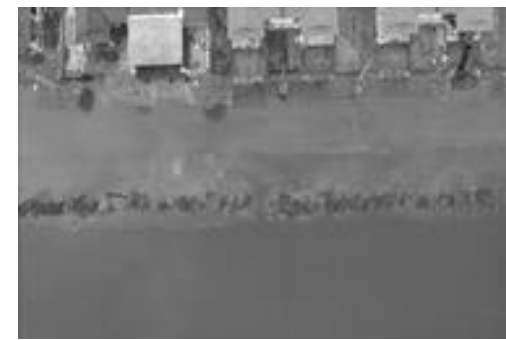

(b)

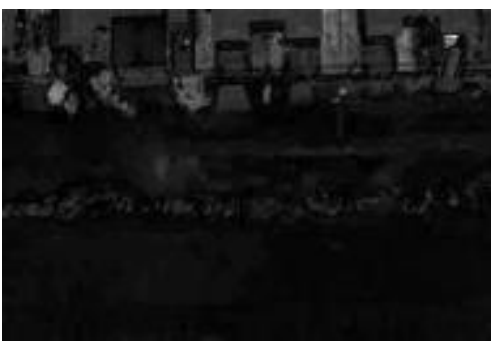

(e)

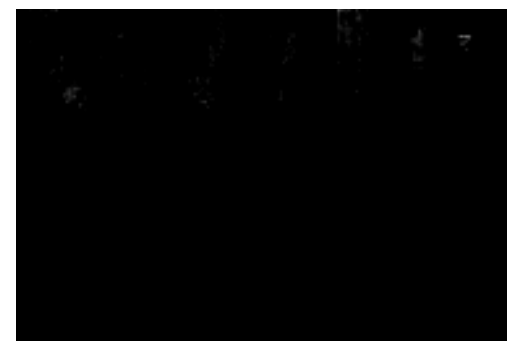

(h)

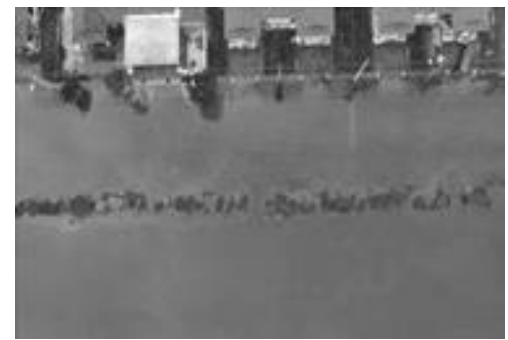

(c)

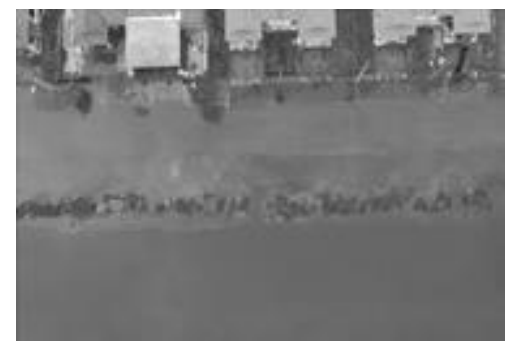

(f)

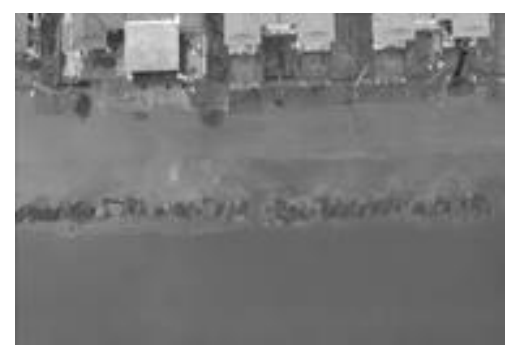

(i)

Figure 3. Visualization of colour channel for RGB, HSI; (a) red channel; (b) green channel; (c) blue channel; (d) hue, H channel, (e) saturation, S channel; (f) intensity, I channel; (g) I+S; (h) I-S and (i) gray level image

Figure 4(a) and (b) gives comparison of segmentation results using k-mean clustering and region growing for each colour channel. From visual observation, there are significant difference in the segmented images of Sample A using k-mean clustering and region gowing technique especially in colour component of red, green, blue, hue, intensity and grey scale. Hence, further image analysis was conducted to determine the segregation accuracy of the images in attempt to deciding which superior colour component will be applied in flood detection formulation.

The overall results of the segmented computations by the use of the region growing and k-mean clustering are presented in Table 1. The result was generated based on 30 sample images and transformed into segmentation process. The results indicated that, the most superior accuracy performance of image segmentation was presented by using Region Growing technique with $88 \%$ that obtained from red colour components. Meanwhile, for k-mean clustering technique the highest only at $77 \%$ accuracy obtained from blue colour components. However, the most concern in this study is about the segmentation process in detecting flooded area. Therefore, since the highest performance from k-mean and region growing has a relatively significant difference, based on their accuracy in detection red colour component from RGB have been taken into account in this study to be used as the suitable technique in flood detection. The mainly reason of the selection is based on their quantitative performances and ability in detection of segmented images. 


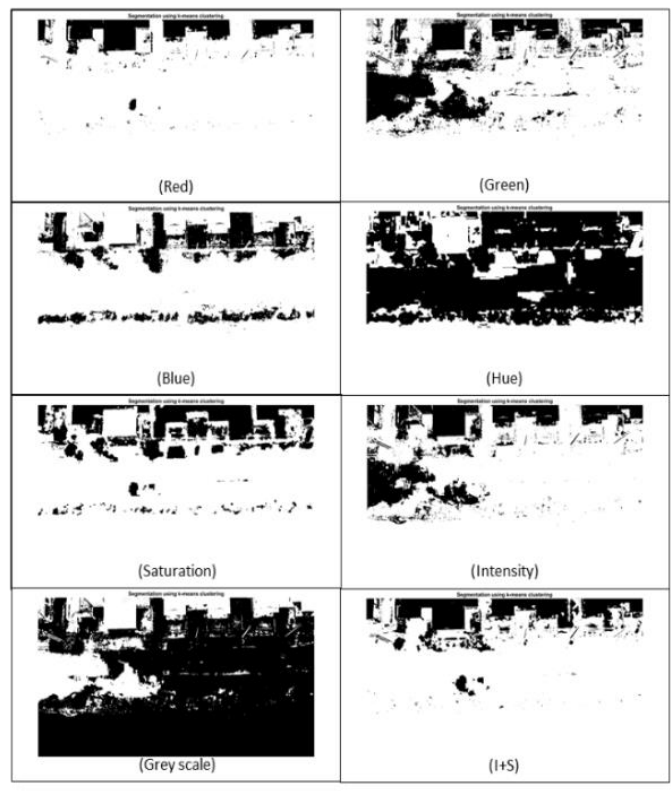

(a)

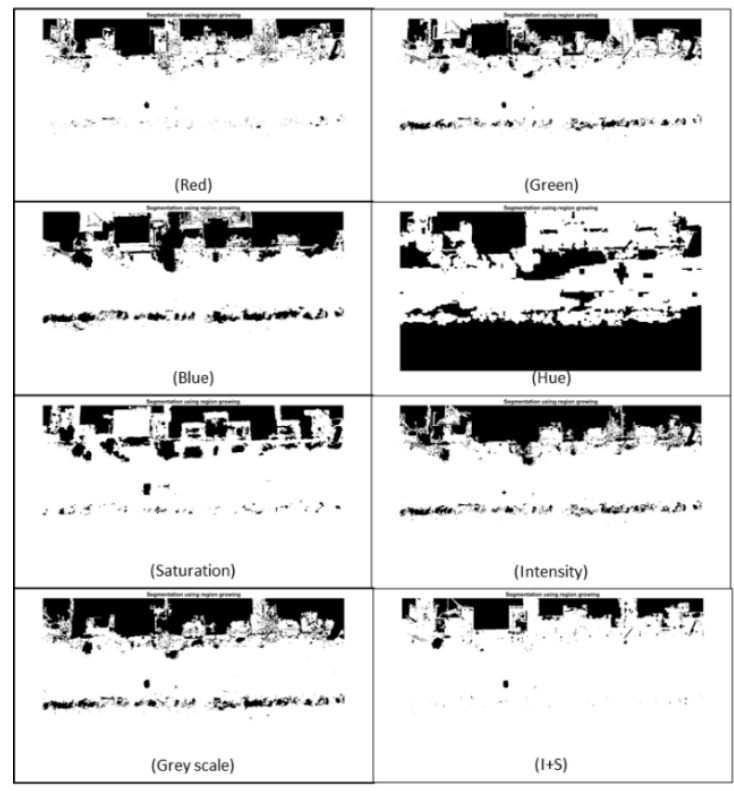

(b)

Figure 4. Comparison of segmentation results using; (a) k-mean clustering and (b) region growing for each colour channel

Table 1. Accuracy and precision of UAV images using different segmentation technique

\begin{tabular}{ccccc}
\hline Colour Components & \multicolumn{2}{c}{ K-Mean Clustering } & \multicolumn{2}{c}{ Region Growing } \\
& Accuracy $(\%)$ & Precision $(\%)$ & Accuracy $(\%)$ & Precision $(\%)$ \\
\hline R & 76.537 & 94.143 & 88.307 & 92.048 \\
G & 73.832 & 91.128 & 84.728 & 91.297 \\
B & 77.399 & 94.365 & 82.842 & 92.875 \\
H & 68.880 & 75.708 & 67.253 & 86.091 \\
S & 67.237 & 69.485 & 66.390 & 93.728 \\
I & 75.811 & 93.380 & 81.572 & 88.359 \\
I+S & 68.248 & 87.929 & 80.140 & 91.180 \\
Grey & 72.962 & 91.266 & 87.706 & 95.322 \\
\hline
\end{tabular}

As stated in previous section about supervised segmentation, the performances in Table 1 are compared against manual segmentation method which is considered as gold standard in attempt to evaluate the segmentation performances. So, the most whichever value that close to the gold standard considers as the good performances assessments. In terms of colour component, the results showed that grey scale and RGB were the most preferable colour component than HSI. It was difficult to yield a high classification accuracy using only the RGB features because the different ground objects may have similar colour. In addition, the water acts as a specular reflector which become the main problem for water detection using aircraft image. It will slow down the detection process to identify the flood areas because of the shadow and bright clear water [5].

\subsection{Flood detection structure}

The flood detection structure was developed based on the segmentation algorithm with the highest accuracy. Hence, Region Growing segmentation technique with RGB colour component was used as the basis for FDS. For validation, a sets of flood images (sample are 1 and sample area 2) were evaluated using the proposed algorithm and the results are presented in Figure 5. From the image of sample area 1, highest FDS value was obtained $66 \%$ using the colour model I+S composition. Meanwhile, the segmented image from red colour model, R of RGB achieved a FDS value of $62.6776 \%$. Both of colour models were able to detect class raw image as flooded image. For the sample area 2, colour model I+S composition has achieved a FDS value of $86 \%$ and FDS value of $77 \%$ was obtained using the red colour model. From validation results, it can be concluded that the proposed algorithm using region growing segmentation technique has successfully detected the flood in the images (FDS value $>50 \%$ ). 


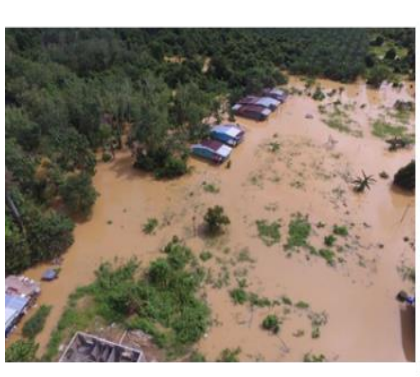

Sample area 1

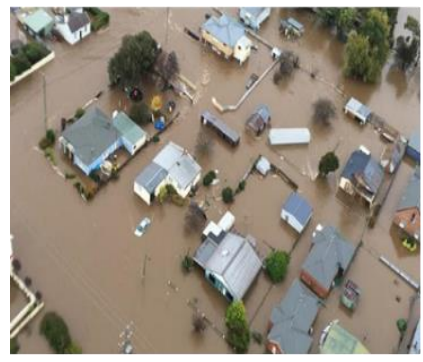

Sample area 2

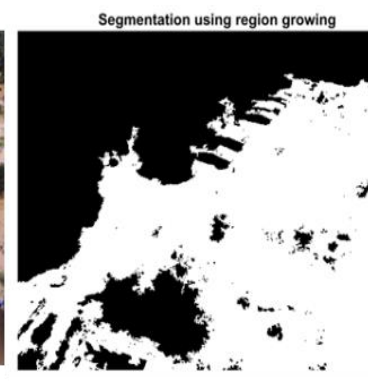

$\mathrm{I}+\mathrm{S}$

Flooded Detection : $66.0967 \%$

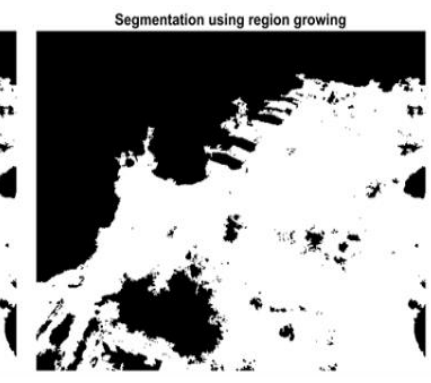

$\mathrm{I}+\mathrm{S}$

Flooded Detection : 62.6776\%

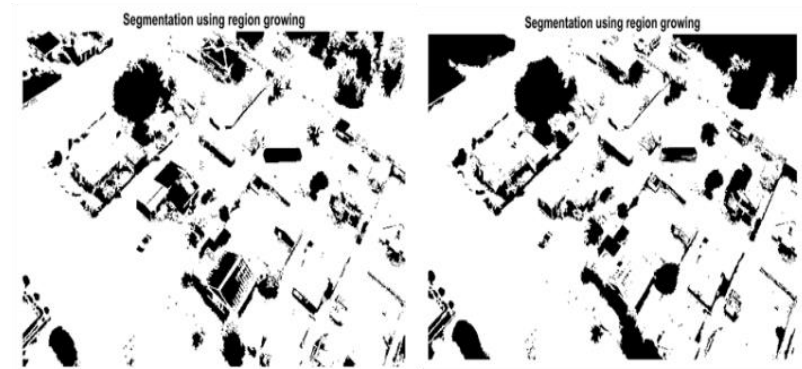

Red of RGB

Flooded Detection : $77.4799 \%$

Figure 5. Percentage of flood discovery using region growing segmentation technique

\section{CONCLUSION}

The application of segmented images from UAV as flood detection area was investigated in this study. The digitalized images that were captured using UAV and analysed using created algorithm using KMean Clustering and Region Growing techniques. The algorithm developed in this study is applicable to be use as flood detection in selected area with reasonable accuracy. The images were analysed to ensure that the input images captured using UAV is more detail and clear as early detection of flood hazard and being a warning alarm in order to reduce the possible disaster in real time. This method can be used as a quantitative tool to measure the extent of flood catastrophe, not only focused on qualitative or visual surveillances detection. A limitation of this work is based on data from a single flood event. Even though the large area of the flood scene was studied, the algorithm needs to be tested further using other events.

\section{ACKNOWLEDGEMENTS}

This research work is supported by University Sultan ZainalAbidin (UniSZA) under grant RAGS (RAGS/1/2015/SG0/Unisza/02/1).

\section{REFERENCES}

[1] Q. Feng, J. Liu, and J. Gong, "Urban flood mapping based on unmanned aerial vehicle remote sensing and random forest classifier-A case of yuyao, China," Water (Switzerland), vol. 7, no. 4, pp. 1437-1455, 2015, doi: $10.3390 /$ w7041437.

[2] J. Thielen, P. Salamon, T. De Groeve, and G. R. Brakenridge, "Evaluation of the satellite-based Global Flood Detection System for measuring river discharge : influence of local factors," Hydrology and Earth System Sciences, vol. 18, no. 11, pp. 4467-4484, 2014, doi: 10.5194/hess-18-4467-2014.

[3] K. E. Joyce, S. E. Belliss, S. V. Samsonov, S. J. McNeill, and P. J. Glassey, "A review of the status of satellite remote sensing and image processing techniques for mapping natural hazards and disasters," Prog. Phys. Geogr., vol. 33, no. 2, pp. 183-207, 2009, doi: 10.1177\%2F0309133309339563.

[4] S. V. Skakun, N. Kussul, and A. Yu Shelestov, "Flood Hazard and Flood Risk Assessment Using a Time Series of Satellite Images: A Case Study in Namibia," Risk Anal., vol. 34, no. 8, pp. 1521-1537, 2014, doi: $10.1111 /$ risa.12156.

[5] D. C. Mason, I. J. Davenport, J. C. Neal, G. J. P. Schumann and P. D. Bates, "Near real-time flood detection in urban and rural areas using high-resolution synthetic aperture radar images," IEEE Trans. Geosci. Remote Sens., vol. 50, no. 8, pp. 3041-3052, 2012, doi: 10.1109/TGRS.2011.2178030. 
[6] A. Alsarayreh and F. S. Mohamad, "Constrained Local Models (CLM) For Facial Feature Extraction using CLNF and SVR as Patch Experts," International Journal of Recent Technology and Engineering, vol. 9, no. 2, pp. 40-43, 2020, doi: 10.1109/ICCVW.2013.54.

[7] V. Klemas, "Coastal and Environmental Remote Sensing from Unmanned Aerial Vehicles : An Overview," Journal of Coastal Research, vol. 315, no. 5, pp. 1260-1267, 2015, doi: 10.2112/JCOASTRES-D-15-00005.1.

[8] A. Sumalan, D. Popescu, M. Ieee, L. Ichim, and M. Ieee, "Flooded area detection using UAV images," 24th Telecommun. forum TELFOR 2016 Serbia, no. 313, pp. 2-5, 2016, doi: 10.1109/TELFOR.2016.7818798.

[9] S. Asrat, "Urban Flood Response Planning: Building Urban Resilience in Calgary and Toronto," Theses and Dissertations, Wilfrid Laurier University, Ontario, Canada, pp. 163, 2015.

[10] O. Kettani and F. Ramdani, "A Fast Deterministic Kmeans Initialization," International Journal of Applied Information Systems., vol. 12, no. 2, 2017, doi: 10.5120/ijais2017451683.

[11] N. Valliammal, "Leaf Image Segmentation Based On the Combination of Wavelet Transform and K Means Clustering," International Journal of Advanced Research in Artificial Intelligence, vol. 1, no. 3, pp. 37-43, 2012, doi: 10.14569/IJARAI.2012.010307.

[12] D. Popescu, L. Ichim, and F. Stoican, "Unmanned Aerial Vehicle Systems for Remote Estimation of Flooded Areas Based on Complex Image Processing," Sensors, vol. 17, no. 3, pp. 446, 2017, doi:10.3390/s17030446.

[13] J. Langhammer and T. Vacková, "Detection and Mapping of the Geomorphic Effects of Flooding Using UAV Photogrammetry," Pure Appl. Geophys, vol. 175, pp. 3223-3245, 2018, doi: 10.1007/s00024-018-1874-1.

[14] G. Ang, W. Shiqiang, W. Fangfang, W. Xiufeng, X. Peng, Y. Lei, and Z. Senlin, "A Newly Developed Unmanned Aerial Vehicle (UAV) Imagery Based Technology for Field Measurement of Water Level," Water, vol. 11, no. 1, pp. 124, 2019, doi: 10.3390/w11010124.

[15] J. Varshali, V. Sharma, and S. Varma, "MMFO: modified moth flame optimization algorithm for region based RGB color image segmentation," International Journal of Electrical and Computer Engineering (IJECE), vol. 10, no. 1, pp. 196-201, 2020, doi: 10.11591/ijece.v10i1.pp196-201.

[16] N. A. Muhadi, A. F. Abdullah, S. K. Bejo, M. R. Mahadi, and A. Mijic, "Image Segmentation Methods for Flood Monitoring System," Water, vol. 12, no. 6, p. 1825, 2020, doi: 10.3390/w12061825.

[17] L. Ichim and D. Popescu, "Segmentation of Vegetation and Flood from Aerial Images Based on Decision Fusion of Neural Networks," Remote Sensing, vol. 12, no. 15, p. 2490, 2020, doi: 10.3390/rs12152490.

[18] X. Zhu, J. Liang and A. Hauptmann, "MSNet: A Multilevel Instance Segmentation Network for Natural Disaster Damage Assessment in Aerial Videos," Proceedings of the IEEE/CVF Winter Conference on Applications of Computer Vision (WACV), 2021, pp. 2023-2032.

[19] A. A. Gebrehiwot and L. Hashemi-Beni, "Three-Dimensional Inundation Mapping Using UAV Image Segmentation and Digital Surface Model," ISPRS Int. Journal of Geo-Information, vol. 10, no. 3, pp. 144, 2021, doi: 10.3390/ijgi10030144.

[20] N. S. Kamaruddin, M. Makhtar, S. N. W. Shamsuddin, and S. A. Fadzli, "Shape-based single object classification using ensemble method classifiers," International Journal on Advanced Science Engineering Information Technology, vol. 7, no. 5, pp. 1908-1912, 2017, doi:10.18517/ijaseit.7.5.1340.

[21] M. K. Osman, M. Y. Mashor, H. Jaafar, R. A. A. Raof, and N. H. Harun, "Performance comparison between RGB and HSI linear stretching for tuberculosis bacilli detection in Ziehl-Neelsen tissue slide images," IEEE International Conference on Signal and Image Processing Applications, pp. 357-362, 2009, doi: 10.1109/ICSIPA.2009.5478677.

[22] I. N. Anida and A. R. Salisa, "Driving cycle development for Kuala Terengganu city using k-means method," International Journal of Electrical and Computer Engineering (IJECE), vol. 9, no. 3, pp. 1780-1787, 2019, doi: 10.11591/ijece.v9i3.pp1780-1787.

[23] S. Kamdi and R. K. Krishna, "Image Segmentation and Region Growing Algorithm," Int. J. Comput. Technol. Electron. Eng., vol. 2, no. 1, pp. 2249-6343, 2012.

[24] S. Roy and S. Bandyopadhyay, "Detection and Quantification of Brain Tumor from MRI of Brain and it 's Symmetric Analysis," Int. J. of Information and Communication Tech. Research, vol. 2, no. 6, pp. 477-483, 2012.

[25] T. Matsuyama, "Expert systems for image processing: Knowledge-based composition of image analysis processes," Comput. Vision, Graph. Image Process., vol. 48, no. 1, pp. 22-49, 1989, doi: 10.1016/0734-189X(89)90103-5.

[26] H. Zhang, J. E. Fritts and S. A. Goldman, "Image segmentation evaluation : A survey of unsupervised methods," Comput. Vis. Image Underst., vol. 110, pp. 260-280, 2007.

[27] R. Khan, A. Hanbury, J. Stottinger and A. Bais, "Color Based Skin Classification," Pattern Recognition Letters, vol. 33, no. 2, pp. 157-163, 2012, doi: 10.1016/j.patrec.2011.09.032.

[28] S. Roberts, "What's the Difference Between the Saturation and the Intensity of a Color?," Quora, 2018. 